\title{
Superuniversality in phase-ordering disordered ferromagnets
}

\author{
Malte Henkel ${ }^{1}$ and Michel Pleimling ${ }^{2}$ \\ ${ }^{1}$ Laboratoire de Physique des Matériaux (LPM), ${ }^{*}$ Nancy Université CNRS, BP 239, F-54506 Vandouvre lès Nancy Cedex, France \\ ${ }^{2}$ Department of Physics, Virginia Polytechnic Institute and State University, Blacksburg, Virginia 24061-0435, USA
}

(Received 8 July 2008; revised manuscript received 25 September 2008; published 29 December 2008)

\begin{abstract}
The phase-ordering kinetics of the ferromagnetic two-dimensional Ising model with uniform bond disorder is investigated by intensive Monte Carlo simulations. Simple aging behavior is observed in the single-time correlator and the two-time responses and correlators. The dynamical exponent $z$ and the autocorrelation exponent $\lambda_{C}$ only depend on the ratio $\varepsilon / T$, where $\varepsilon$ describes the width of the distribution of the disorder, whereas a more complicated behavior is found for the nonequilibrium exponent $a$ of the two-time response as well as for the autoresponse exponent $\lambda_{R}$. The scaling functions are observed to depend only on the dimensionless ratio $\varepsilon / T$. If the length scales are measured in terms of the time-dependent domain size $L(t)$, the form of the scaling functions is in general independent of both $\varepsilon$ and $T$. Conditions limiting the validity of this "superuniversality" are discussed.
\end{abstract}

DOI: 10.1103/PhysRevB.78.224419

PACS number(s): 75.10.Nr, 64.60.Ht, 05.70.Ln

\section{INTRODUCTION}

Aging phenomena have become one of the paradigmatic examples which are used to study fundamental aspects of nonequilibrium criticality, besides their practical use in materials processing. If some physical system is brought rapidly out of equilibrium by a sudden change in an external control parameter (an often used device is to quench the system by lowering its temperature rapidly from the disordered hightemperature phase to its ordered low-temperature phase where at least two thermodynamically stable states coexist) one often finds simultaneously the following three properties: (i) slow (i.e., nonexponential) dynamics, (ii) breaking of time-translation invariance, and (iii) dynamical scaling, which together are said to constitute aging behavior. These features were first observed together in the mechanical properties of many polymeric materials by Struik, ${ }^{1}$ and it has since been understood that the broad characteristics of aging can be found in many different types of nonequilibrium systems. Besides spin glasses, ${ }^{2,3}$ other well-studied examples may be found in the phase-ordering kinetics of a ferromagnet quenched below its critical temperature $T_{c}>0$ (Ref. 4) or in granular media. ${ }^{5}$

The analysis of phase ordering as it occurs in ferromagnets quenched from an initially disordered state into its coexistence phase with temperature $T<T_{c}$ is particularly simple to formulate. The motion of the domain walls is driven by the surface tension between the ordered domains. The typical time-dependent length scale is related to the linear size of these ordered domains which grows as $L=L(t)$ $\sim t^{1 / z}$, where $z$ is the dynamical exponent. For a nonconserved order parameter, it is well known that $z=2 .{ }^{4}$ Because of the simple algebraic scaling of the linear domain size $L(t)$, one expects the following scaling behavior for the singletime correlation function,

$$
C(t ; \boldsymbol{r}):=\langle\phi(t, \boldsymbol{r}) \phi(t, \mathbf{0})\rangle=t^{-b} F\left(\frac{|\boldsymbol{r}|}{L(t)}\right),
$$

for sufficiently large times $t \gg t_{\text {micro }}$ [with a microscopic reference time $t_{\text {micro }}$ such that $L\left(t_{\text {micro }}\right)$ is of the order of the lattice constant]. Similarly, for the two-time correlation and response functions (in the aging regime, where the observation time $t$ and the waiting time $s$ satisfy $t, s \gg t_{\text {micro }}$, and $\left.t-s \gg t_{\text {micro }}\right)$ :

$$
\begin{gathered}
C(t, s ; \boldsymbol{r}):=\langle\phi(t, \boldsymbol{r}) \phi(s, \mathbf{0})\rangle=s^{-b} F_{C}\left(\frac{t}{s}, \frac{|\boldsymbol{r}|}{L(s)}\right), \\
R(t, s ; \boldsymbol{r}):=\left.\frac{\delta\langle\phi(t, \boldsymbol{r})\rangle}{\delta h(s, \mathbf{0})}\right|_{h=0}=s^{-1-a} F_{R}\left(\frac{t}{s}, \frac{|\boldsymbol{r}|}{L(s)}\right) .
\end{gathered}
$$

Here $\phi(t, r)$ is the space-time-dependent order parameter, whereas $h(s, r)$ is the conjugate magnetic field (spatial translation invariance of all averages will be assumed throughout this paper) and $a$ and $b$ are aging exponents. The scaling functions $F_{C, R}(y, \mathbf{0}) \sim y^{-\lambda_{C, R} / z}$ for $y \rightarrow \infty$ which defines the autocorrelation exponent $\lambda_{C}$ and the autoresponse exponent $\lambda_{R}$. For phase-ordering kinetics in pure systems, it is generally admitted that $b=0$ and simple scaling arguments show that $a=1 / z$. For an initial high-temperature state and for pure ferromagnets, $\lambda_{C}=\lambda_{R}$ is independent of the known equilibrium exponents. ${ }^{2,4,6-10}$ The scenario just described is referred to as simple aging. The conditions for the onset of aging and the possible scaling forms have been carefully discussed in Refs. 11 and 12 .

In going from phase-ordering kinetics in simple ferromagnets to glassy systems (usually modeled by spin systems with disorder and frustration), one expects more complicated growth laws $L=L(t)$ which describe a crossover from the domain growth of essentially pure systems [as long as $L(t)$ is small compared to the typical distance between disordercreated defects] to a late-time regime with a slower growth and dominated by the defect structure. For disordered ferromagnets without frustration, this can be studied through generalizations of the Allen-Cahn equation, which attempt to describe how the pinning of the domain walls created by the disorder should be overcome by thermal activation. ${ }^{13,14}$ In addition, it was suggested that once the unique reference length scale is chosen to be $L(t)$, the resulting scaling functions should become superuniversal in the sense that they 
should be independent of the disorder. ${ }^{15}$ This superuniversality has indeed been confirmed for the single-time spin-spin correlator. ${ }^{16-21}$ Furthermore, superuniversality is in qualitative agreement with the experimental observation in several distinct polymers and metals that the linear response to a small mechanical stress can be described in terms of an universal master curve which is independent of the material studied. ${ }^{1}$ The universal scaling functions of the pure case can be calculated from the theory of local scale invariance. ${ }^{22}$ Tests in nonintegrable systems include the auto- and spacetime responses in the two-dimensional (2D) and threedimensional Ising models, ${ }^{23}$ the autocorrelation function in the two-dimensional Ising model,${ }^{24}$ the same quantities in the two-dimensional $q$-states Potts model with $q=2,3$, and 8 (Ref. 25) as well as the autoresponse function in several other cases; see Ref. 26 for a recent review. Therefore, with the help of superuniversality, if confirmed, the scaling functions describing nonequilibrium relaxation of quite complex systems would become analytically treatable.

In this work, we shall consider a two-dimensional ferromagnetic Ising model with quenched bond disorder. The nearest-neighbor Hamiltonian is given by ${ }^{27,28}$

$$
\mathcal{H}=-\sum_{(i, j)} J_{i j} \sigma_{i} \sigma_{j}, \quad \sigma_{i}= \pm 1 .
$$

The random variables $J_{i j}$ are uniformly distributed over $[1-\varepsilon / 2,1+\varepsilon / 2]$, where $0 \leq \varepsilon \leq 2$. The model has a secondorder phase transition at a critical temperature $T_{c}(\varepsilon)>0$ between a paramagnetic state and a ferromagnetic state. It is thought that $T_{c}(\varepsilon) \approx T_{c}(0) \simeq 2.269 \ldots$ should not depend strongly on $\varepsilon$. Using heat-bath dynamics with a nonconserved order parameter and starting from a fully disordered initial state, phase ordering occurs, and there is evidence which suggests that the characteristic length scale $L=L(t)$ $\sim t^{1 / z}$ should scale algebraically and where the dynamical exponent $z=z(T, \varepsilon)$ should depend continuously on the temperature and the disorder $\varepsilon$. Indeed, generalizing the HuseHenley heuristic argument ${ }^{13}$ by considering the case when the disorder-created energy barriers for the motion of the domain walls are distributed logarithmically with respect to the domain size $L(t)$, the form 27,29

$$
z=z(T, \epsilon) \stackrel{?}{=} 2+\epsilon / T
$$

was proposed, where the constant $\epsilon$ parametrizes the barrier height. Simulations of the linear domain size ${ }^{27}$ seemed to confirm this, with the empirical identification $\epsilon=\varepsilon$ and are also consistent with the results of field-theoretical studies in the Cardy-Ostlund model. ${ }^{30}$ Data from the thermoremanent magnetization $M_{\mathrm{TRM}}(t, s)=h \int_{0}^{s} d u R(t, u)=s^{-a} f_{M}(t / s)$ were used to estimate the exponent $a$ and, assuming $a=1 / z$, also looked consistent with Eq. (5) and $\epsilon=\varepsilon$, at least for values for $T$ and $\varepsilon$ for which $z$ did not become too large. ${ }^{31}$ Superuniversality has been confirmed recently for the hull enclosed area. ${ }^{32}$ On the other hand, the conclusion of a simple aging reached in Refs. 27-29 has been questioned by more recent simulations for the random-site Ising model. ${ }^{33}$ In that work, a scaling form $C(t, s)=C_{\mathrm{st}}(t-s)+C_{\mathrm{ag}}[h(t-s) / h(s)]$ with $h(u)=\exp \left(\frac{u^{1-\mu}}{1-\mu}\right)$ was considered, where $\mu$ is a fit param- eter. In the limit $\mu \rightarrow 1$, one recovers the simple aging scenario described above [the stationary part $C_{\mathrm{st}}(t-s)$ merely represents an irrelevant correction to the leading scaling behavior]. The case $\mu<1$ is called subaging, and the case $\mu>1$ is called superaging. In Ref. 33 systematic deviations from the dynamical scaling of simple aging were observed in the $2 \mathrm{D}$ random-site Ising model quenched to below $T_{c}$. A data collapse could be achieved, however, by allowing $\mu$ to vary, and values in the range $\mu \approx 1.03-1.04$ were reported. ${ }^{33}$ This finding was interpreted as to suggest the presence of a slight superaging effect. ${ }^{33}$

In the following, we shall present data on the single-time and two-time correlations as well as on two-time response functions. As we shall see in Sec. II, our data are fully compatible with the simple aging scenario, and furthermore, looking at a larger range of values of $z$, we find that the dynamical exponent $z=z(T, \varepsilon)=z(\varepsilon / T)$ depends on the control parameters in a more complicated way than that suggested in Eq. (5). These conclusions are also valid for the two-time response function. The dependence of the various nonequilibrium exponents on both $\varepsilon$ and $T$ will be studied. We also show evidence that the scaling functions only depend on the ratio $\varepsilon / T$ of the control parameters and finally confirm the generic superuniversality of the scaling functions of correlation and response functions. However, we also find two conditions which must be satisfied for superuniversality to hold. Our conclusions are given in Sec. III.

\section{RESULTS}

The simulations are carried out as follows. For the integrated response we simulated systems with $\mathcal{N}=300 \times 300$ spins using the standard heat-bath algorithm. Prepared in an uncorrelated initial state corresponding to infinite temperatures, the system is quenched to the final temperature in the presence of a random binary field $h_{i}= \pm h$, with strength $h$ $=0.05$, following the well-established method of Barrat $^{34}$ (using a random field avoids a bias which would drive the system rapidly out of the scaling regime). Turning off the random field after a waiting time $s$, the thermoremanent magnetization $M_{\mathrm{TRM}}(t, s)=\frac{1}{T \mathcal{N}} \Sigma_{i} \overline{\left\langle\sigma_{i}(t) h_{i}(s)\right\rangle}$ is measured at time $t$. We averaged over at least $5 \times 10^{4}$ different runs with different initial states and different realizations of the noise. We point out that the data discussed in this paper are of much higher quality than our earlier data ${ }^{31}$ for the autoresponse. For the autocorrelation function, we considered systems with up to $600 \times 600$ spins in order to avoid the appearance of finite-size effects for the times accessed in the simulations. The data discussed in the following have been obtained after averaging over at least 5000 different runs with different random numbers. Our main focus was on $\varepsilon=0.5,1$, and 2 where we considered for every case at least four different temperatures. In addition, some runs where also done for other values of $\varepsilon$. The total study took approximately $2 \times 10^{5}$ CPU hours on Virginia Tech's System X supercomputer composed of Dual $2.3 \mathrm{GHz}$ PowerPC 970FX processors.

Our first question is about the scaling form to be used. In Fig. 1, we show data for the autocorrelation $C(t, s)$ 

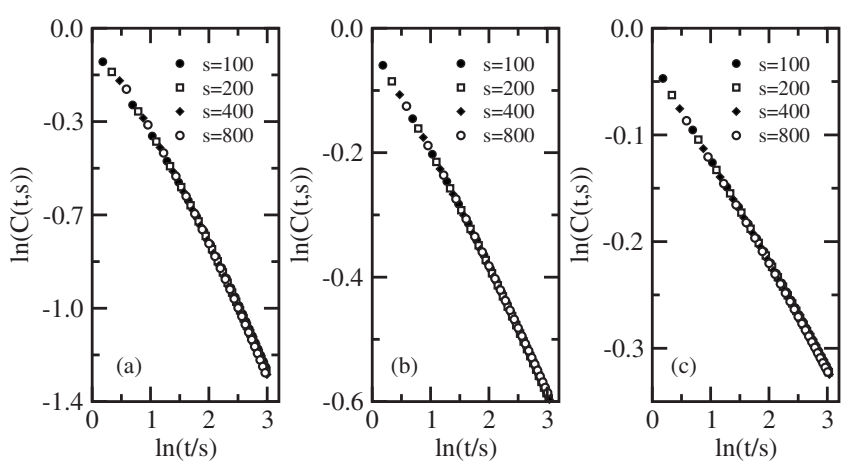

FIG. 1. Scaling of the autocorrelation $C(t, s)$ for (a) $\varepsilon=0.5$ and $T=1$, (b) $\varepsilon=1$ and $T=0.4$, and (c) $\varepsilon=2$ and $T=0.4$, for several values of $s$. Here and in the following error bars are smaller than the sizes of the symbols, unless explicitly stated otherwise.

$=\mathcal{N}^{-1} \sum_{i}\left\langle\sigma_{i}(t) \sigma_{i}(s)\right\rangle$, plotted over against $t / s$, for several typical values of $\varepsilon$ and $T$. A nice data collapse is seen, which is fully consistent with simple aging. Our scaling plots also imply that the exponent $b=0$, analogously to what is found in the phase ordering of pure systems. In Ref. 33, a superaging scaling form $C(t, s)=\mathcal{C}\left(\exp \left[\frac{(t-s)^{1-\mu_{-} s^{1-\mu}}}{1-\mu}\right]\right)$ was considered for the random-site Ising model where the exponent $\mu>1$ is fitted to the data. Simple aging is recovered in the $\mu \rightarrow 1$ limit. However, the values of $\mu \approx 1.03$ reported in Ref. 33 are so close to unity that a careful study on possible finitetime corrections to scaling appears to be required before such a conclusion could be accepted. ${ }^{35}$ For all $\varepsilon<2$, our data for the random-bond model show no hint for a superaging behavior, ${ }^{36,37}$ in contrast to the findings in Ref. 33. Note that the observed scaling form of simple aging would be incompatible with a non-power-law form of $L(t)$ in the range of times considered. We also remark that for $\varepsilon=2$ and larger temperatures finite-time corrections to simple aging are observed; see Refs. 31 and 38.

Having in this way checked that the relevant length scale $L=L(t)$ should indeed scale algebraically with time, we next determined the dynamical exponent $z=z(T, \varepsilon)$ from the criterion $^{32}$ involving the single-time correlator,

$$
C[t, L(t)] \stackrel{!}{=} \frac{1}{2}, \quad L(t) \sim t^{1 / z(T, \varepsilon)}
$$

The results for $z$ are shown in Fig. 2 and listed in Table I. First, we observe that the values of $z$ obtained in fact only depend on the dimensionless ratio $\varepsilon / T$, to within our numerical accuracy. Second, we see that the function $z=z(\varepsilon / T)$ is nonlinear and only becomes an approximately linear function in a relatively small region of values of $z$. We are confident that our results are more reliable than earlier ones since they do not just describe the scaling of a single quantity but rather will be needed for the correct scaling description of several other observables, as we shall show below. We stress that only the values of $z$ as given in Table I will lead to a good scaling according to simple aging without having to consider possible corrections to scaling.
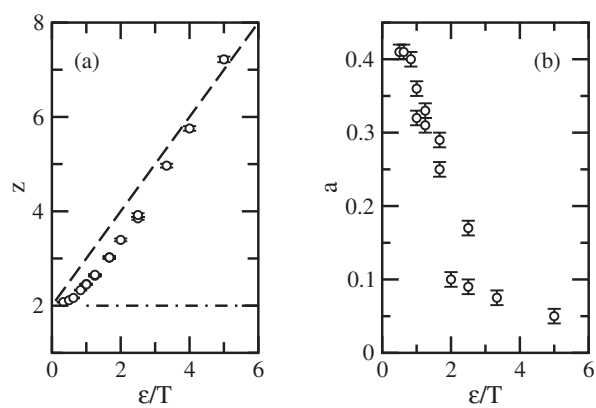

FIG. 2. Left panel: Dynamical exponent $z$, determined from the condition $C[t, L(t)] \stackrel{!}{=} \frac{1}{2}$, as a function of $\varepsilon / T$. The dashed line corresponds to Eq. (5). Right panel: aging exponent $a$, as determined from the scaling of $M_{\mathrm{TRM}}(t, s)$. In some cases we have more than one data point for a given value of $\varepsilon / T$. These data points correspond to different values of $\varepsilon$ and $T$ with $\varepsilon / T$ constant (see Table I) showing that $a$ is not simply a function of the ratio $\varepsilon / T$ but that it depends in a more complicated way on both $\varepsilon$ and $T$.

In the same way, in Fig. 3 we show data for the scaling of the thermoremanent magnetization, expected to be of the form

$$
M_{\mathrm{TRM}}(t, s)=s^{-a} f_{M}(t / s) .
$$

The exponent $a$ is obtained in the usual way ${ }^{39}$ by plotting the thermoremanent magnetization as a function of the waiting time $s$ for fixed values of the ratio $t / s$. The resulting powerlaw decay [see Eq. (7)] then yields the value of the exponent $a$. The numerical values and error bars given in Table I and shown in Fig. 2 are obtained after averaging over the values obtained for five different values of $t / s$, namely, 3, 5, 7, 10, and 15. Looking at these values (see Fig. 2), we observe that the estimates $a=a(T, \varepsilon)$ scatter considerably more than those for $z$. We consider this scatter to be large enough to conclude that $a$ cannot be reduced to a function of the single variable $\varepsilon / T$. Furthermore, considering in detail the numerical values from Table I, we see that the relation $a=1 / z$, known from the phase ordering of pure ferromagnets, ${ }^{2,40,41}$ is no longer valid.

In order to understand this finding, let us briefly reconsider how the relation $a=1 / z$ may be derived for pure ferromagnets. Consider a pure ferromagnet in an external oscillating magnetic field of angular frequency $\omega$. The dissipative part of the linear response is given as the imaginary part of the dynamic susceptibility and reads (see, e.g., Ref. 2),

$$
\chi^{\prime \prime}(\omega, t)=\int_{0}^{t} d u R(t, u) \sin [\omega(t-u)]=\chi_{1}^{\prime \prime}(\omega)+t^{-a} \chi_{2}^{\prime \prime}(\omega t)+\ldots
$$

where the last relation follows from the usually assumed scaling [Eq. (3)] of the autoresponse function $R(t, s)$. On the other hand, motivated from the physical picture that the dynamics in phase ordering should only come from the motion of the domain walls between the ordered domains, one would expect to find ${ }^{40}$ 
TABLE I. Dynamical exponent $z$, nonequilibrium response exponent $a$, the autocorrelation exponent $\lambda_{C} / z$, and the autoresponse exponent $\lambda_{R} / z$ for different values of $\varepsilon$ and $T$.

\begin{tabular}{cccccc}
\hline \hline$\varepsilon$ & $T$ & $z$ & \multicolumn{1}{c}{$a$} & \multicolumn{1}{c}{$\lambda_{C} / z$} & $\lambda_{R} / z$ \\
\hline 0.5 & 1.5 & $2.08(1)$ & & $0.59(1)$ & \\
0.5 & 1.0 & $2.11(1)$ & $0.41(1)$ & $0.565(10)$ & $0.61(2)$ \\
0.5 & 0.8 & $2.16(1)$ & $0.41(1)$ & $0.56(1)$ & $0.61(3)$ \\
0.5 & 0.6 & $2.33(1)$ & $0.40(1)$ & $0.54(1)$ & $0.60(2)$ \\
0.5 & 0.5 & $2.46(1)$ & $0.36(1)$ & $0.48(1)$ & $0.55(2)$ \\
0.5 & 0.4 & $2.64(2)$ & $0.33(1)$ & $0.46(1)$ & $0.50(2)$ \\
0.5 & 0.3 & $3.02(2)$ & $0.29(1)$ & $0.385(10)$ & $0.46(2)$ \\
1.0 & 1.0 & $2.45(1)$ & $0.32(1)$ & $0.49(1)$ & $0.51(2)$ \\
1.0 & 0.8 & $2.65(2)$ & $0.31(1)$ & $0.445(10)$ & $0.50(2)$ \\
1.0 & 0.6 & $3.02(2)$ & $0.25(1)$ & $0.38(1)$ & $0.43(2)$ \\
1.0 & 0.4 & $3.85(3)$ & $0.17(1)$ & $0.29(1)$ & $0.34(1)$ \\
1.5 & 0.9 & $3.02(3)$ & & $0.375(10)$ & \\
2.0 & 1.0 & $3.39(3)$ & $0.10(1)$ & $0.315(10)$ & $0.35(2)$ \\
2.0 & 0.8 & $3.92(4)$ & $0.09(1)$ & $0.270(5)$ & $0.32(2)$ \\
2.0 & 0.6 & $4.97(4)$ & $0.075(10)$ & $0.217(3)$ & $0.28(1)$ \\
2.0 & 0.5 & $5.76(5)$ & & $0.189(3)$ & $0.21(1)$ \\
2.0 & 0.4 & $7.22(6)$ & $0.05(1)$ & $0.155(3)$ & \\
\hline \hline
\end{tabular}

$$
\chi^{\prime \prime}(\omega, t)=\chi_{\mathrm{st}}^{\prime \prime}(\omega)+L(t)^{-1} \chi_{\mathrm{age}}^{\prime \prime}(\omega t)+\ldots
$$

from which one may identify the stationary and the aging part with the terms in Eq. (8) coming from the scaling analysis. Since only the domain boundaries contribute to the dynamics, the leading time-dependent part should be proportional to the surface area of the domain divided by the total volume, hence to $L(t)^{d-1} / L(t)^{d}=1 / L(t)$ which accounts for the factor $1 / L(t)$ in Eq. (9). Comparison of Eqs. (8) and (9), together with $L(t) \sim t^{1 / z}$, then gives $a=1 / z$.

Our empirical observation that $a z<1$ suggests that the above argument should no longer apply to random ferromagnets. Since Eq. (8) only depends on the dynamical scaling assumption [Eq. (3)] and given that our numerical results appear to be compatible with it, we expect that Eq. (8) should remain valid for disordered ferromagnets. Since also for disordered ferromagnets, the contribution to the aging behavior should come from the boundary region between
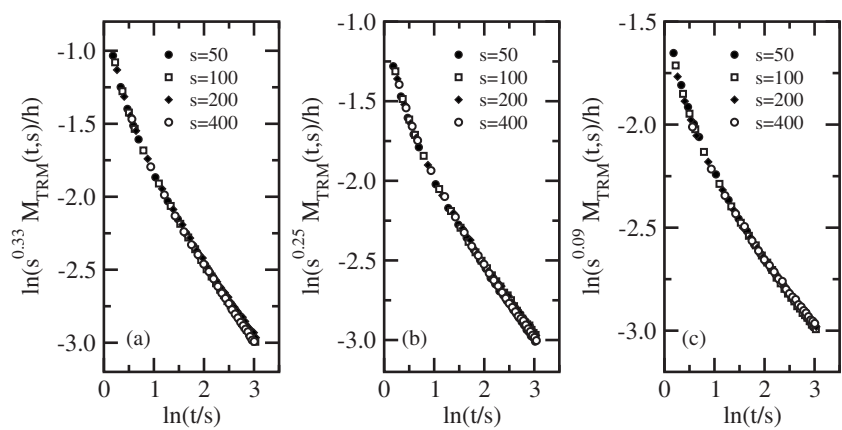

FIG. 3. Scaling of the thermoremanent magnetization $M_{\mathrm{TRM}}(t, s)$ for several values of $s$ with (a) $\varepsilon=0.5$ and $T=0.4$, (b) $\varepsilon=1$ and $T=0.6$, and (c) $\varepsilon=2$ and $T=0.8$. ordered domains (this is also suggested by looking at the microscopic spin configurations, see, e.g., Ref. 38), we expect it to be proportional to $N_{d}(L) / N_{b}(L)$, where $N_{b, d}(L)$ denote the number of mobile spins in the bulk and at the domain boundaries, respectively. While one should still have $N_{b}(L) \sim L^{d}$, disorder may cause the domain boundary to become fractal, and hence, $N_{d}(L) \sim L^{d_{f}}$, with $d_{f}$ the fractal dimension (and $d_{f}=d-1$ for the pure case). Then Eq. (9) would be replaced by

$$
\chi^{\prime \prime}(\omega, t)=\chi_{\mathrm{st}}^{\prime \prime}(\omega)+L(t)^{-\left(d-d_{f}\right)} \chi_{\text {age }}^{\prime \prime}(\omega t)+\ldots,
$$

and comparison with Eq. (8) would now imply

$$
a=\frac{d-d_{f}}{z} .
$$

Our empirical results (Table I) imply that $d_{f}>d-1$, that is, the disorder should modify the domain boundaries into fractal curves. From Eq. (11), since $a$ depends on the dynamical exponent $z=z(\varepsilon / T)$ as well as the fractal dimension $d_{f}$, it may appear more natural that a cannot be written as a function of the single variable $\varepsilon / T$.

In Fig. 4 we show the scaling behavior of the spacedependent and time-dependent correlation and response functions for various values of the waiting time $s$. For the selected typical values of $\varepsilon$ and $T$, a simple aging behavior is observed, in agreement with the observed scaling behavior of the autocorrelation and of the autoresponse. We have found completely analogous results for all other values of $\varepsilon$ and of $T$ which we considered. In fact, the scaling is much cleaner than for the $\boldsymbol{r}=0$ quantities and for none of the studied cases a sizable correction to scaling could be identified. Obviously, space-dependent and time-dependent quantities are much better suited for an investigation of the scaling forms than 

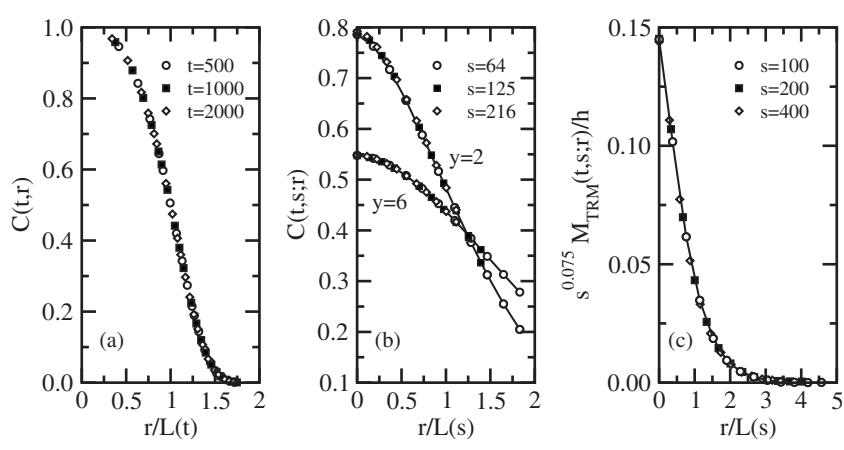

FIG. 4. (a) Scaling behavior of the single-time correlator $C(t, \boldsymbol{r})$ for $\varepsilon=1$ and $T=0.8$ and several values of $t$ as a function of the scaling variable $r / L(t)$. (b) Scaling of the space-time-dependent correlator $C(t, s ; \boldsymbol{r})$ for $\varepsilon=0.5$ and $T=0.5$ for several values of $s$ and two fixed values of $y=t / s$ as a function of the scaling variable $r / L(s)$. (c) Scaling of the space-time-dependent thermoremanent magnetization $M_{\mathrm{TRM}}(t, s ; \boldsymbol{r})$ for $\varepsilon=2$ and $T=0.6$ for several values of $s$, with $y=t / s=5$. The lines are guides for the eyes.

quantities that only depend on time. Similar conclusions have recently been drawn from a study of nonequilibrium growth models. ${ }^{42}$

Using the scaling forms (2) and (3) in the limit of large $y=t / s$ for the autocorrelation and autoresponse functions (where $\boldsymbol{r}=0$ ), we have also extracted the exponents $\lambda_{C} / z$ and $\lambda_{R} / z$ and list our results in Table I. In contrast to the pure case, where for fully disordered initial conditions one may show that $\lambda_{C}=\lambda_{R},{ }^{4,10}$ the values of the autocorrelation exponent $\lambda_{C}$ are different from those of the autoresponse exponent $\lambda_{R}$. In particular, we find that within our numerical accuracy, $\lambda_{C} / z$ is a function of the single variable $\varepsilon / T$, at least for $\varepsilon<2$, while $\lambda_{R} / z$ cannot be expressed in this way. Our data suggest that $\lambda_{R} \geq \lambda_{C}$ and they are consistent with the rigorous Yeung-Rao-Desai inequality $\lambda_{C} \geq d / 2$. ${ }^{43}$ Furthermore, we observe that $\lambda_{R} / z-a$ should be practically constant (again for $\varepsilon<2$ and with a value in the range $\approx 0.17-0.20$ ). In conclusion, our data are clearly consistent with simple aging of the single-time and two-time correlation functions, as well as for the thermoremanent magnetization, and fully confirm the anticipated scaling forms (1)-(3) with $L(t) \sim t^{1 / z}$.

Next, we shall compare the form of the scaling functions, for several values of the control parameters $\varepsilon$ and $T$. Since the dynamical exponent $z=z(\varepsilon / T)$, see Fig. 2, one might expect that the scaling functions themselves should only depend on the ratio $\varepsilon / T$, rather than on $\varepsilon$ and $T$ separately. In Fig. 5, we test this idea by comparing data for $\varepsilon=T=1$ with those for $\varepsilon=T=0.5$, for the three cases of (a) the space-timedependent correlation $C(t, s ; \boldsymbol{r})$, (b) the autocorrelation $C(t, s)$, and (c) the space-time-dependent thermoremanent magnetization $M_{\mathrm{TRM}}(t, s ; \boldsymbol{r})=h \int_{0}^{s} d u R(t, u ; \boldsymbol{r})$. In all cases, there is a clear scaling behavior consistent with simple aging $^{44}$ and the scaling functions nicely superpose (for the integrated response, the data for $\varepsilon=T=0.5$ were multiplied by 1.17 in order to take into account the well-known presence of the nonuniversal numerical prefactor). This result, namely, that the form of the scaling function only depends on the ratio $\varepsilon / T$, goes beyond the standard scaling forms (1)-(3), yet it does not require to rescale the length by the
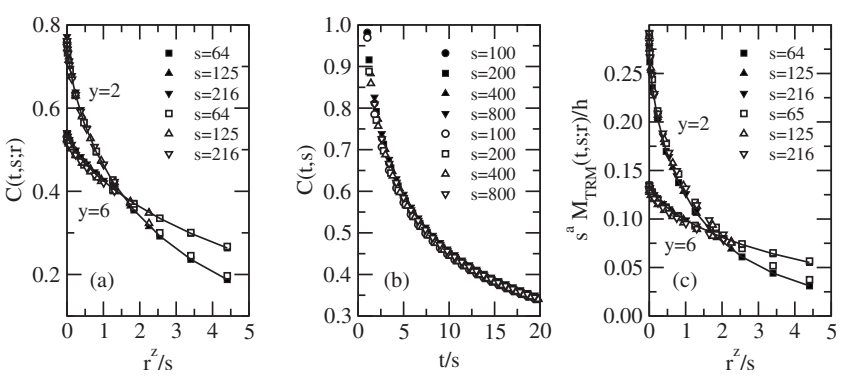

FIG. 5. (a) Space-time correlator $C(t, s ; \boldsymbol{r})$ for $\varepsilon=T=1$ (filled symbols) and $\varepsilon=T=0.5$ (open symbols) for several waiting times $s$ and the values $y=t / s=2$ and $y=t / s=6$. The full curves are guides for the eyes. (b) Autocorrelator $C(t, s)$ for $\varepsilon=T=1$ (filled symbols) and $\varepsilon=T=0.5$ (open symbols) for several waiting times $s$. (c) Scaled space-time thermoremanent magnetization for $\varepsilon=T=1$ (filled symbols) (with $a=0.32$ ) and $\varepsilon=T=0.5$ (open symbols) (with $a=0.36$ ) for several waiting times $s$ and the values $y=t / s=2$ and $y=t / s=6$. The full curves are guides for the eyes.

typical domain size $L(t)$, as it would be required for a test of superuniversality. More systematic tests of this result would be welcome. We point out that the findings of Fig. 5 are consistent with our earlier observation that $\lambda_{C}=\lambda_{C}(\varepsilon / T)$. The more complicated dependence of $\lambda_{R}$ on both $\varepsilon$ and $T$ would only appear if in plots such as Fig. 5(c) one would concentrate on the region $|\boldsymbol{r}| \approx 0$.

After these preparations, we are ready for a test of superuniversality. Superuniversality of the single-time correlator $C(t, r)$ is tested in Fig. 6. First, we show in Fig. 6(a) data for several values of $0 \leq \varepsilon<2$ and $T$, where the values of $z$ may be read off from Fig. 2 and Table I. The times were chosen sufficiently large such that a clear scaling behavior has set in. Using the typical length scale $L(t)$ as determined earlier from Eq. (6), we see that indeed all data, including the one for the case without disorder, collapse nicely onto a single curve, within the numerical accuracy. This is a clear confirmation of superuniversality, very much in agreement with earlier studies. ${ }^{16-21,32}$ However, when considering the case $\varepsilon=2$, which is shown in Fig. 6(b), a different picture emerges. Clearly, the scaling curves for $C(t, r)$ as obtained for several values of $T$ again collapse onto each other, but, as the com-
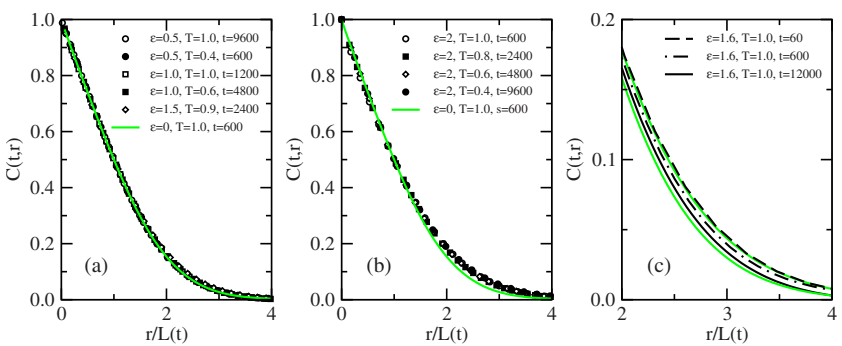

FIG. 6. (Color online) Test of the superuniversality for the single-time correlation function. In panel (a), data for $\varepsilon=0,0.5,1$, and 1.5 are shown to fall onto the same rescaled curve. In panel (b), we show that the data for $\varepsilon=2$ describe a curve different from the one of panel (a). The gray curve is the scaling function of the pure system. In panel (c), data for $\varepsilon=1.6$ and different times are compared with the scaling curves as measured for $\varepsilon=0$ (lower gray line) and $\varepsilon=2$ (upper gray line). 

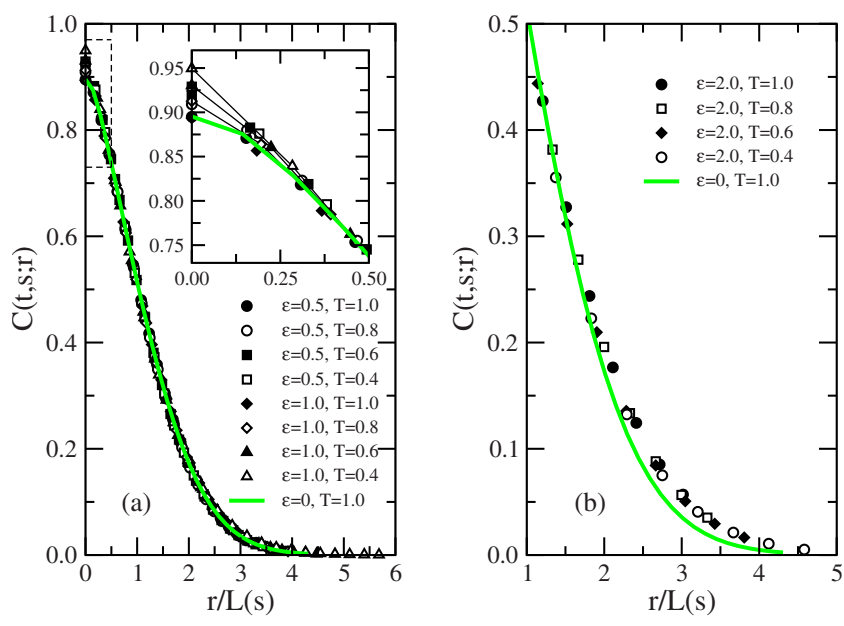

FIG. 7. (Color online) Test of the superuniversality of the twotime correlation function with $y=t / s=4$ and analogous to Fig. 6 . The data shown are for $s=100$. The gray curves show the data of the pure system. The inset in panel (a) is a magnification of the area within the dashed box. The black lines are guides for the eyes.

parison with the scaling function of the pure case shows, the scaling function is no longer the same as the one of the pure case. Therefore, if taken at face value, the case $\varepsilon=2$ might represent a distinct superuniversality class. In order to get a better understanding on this point, we show in Fig. 6(c) data for a relatively large value of $\varepsilon$. One sees that for moderately large times, the data are very close to the curve found for $\varepsilon=2$, but when the time $t$ is made very large, a crossover to the curve of the pure case $\varepsilon=0$ is observed. Qualitatively, the crossover time $t_{\times}$increases when $\varepsilon \rightarrow 2$ and becomes so large that a crossover is no longer detectable for the times (and the lattice sizes, which must be increased for large times in order to avoid finite size effects) reachable with our numerical methods. We did not see any sign for a crossover in our data with $\varepsilon=2$, but purely numerical techniques cannot distinguish between a very large and an infinite crossover time $t_{\times}$.

We extend the test of superuniversality to the case of space-time-dependent two-time correlators in Fig. 7 and similarly for the space-time-dependent two-time response in Fig. 8. Qualitatively, we arrive at essentially the same conclusion as for the single-time correlator. In both cases, panel (a) demonstrates a superuniversal behavior for values of 0 $\leq \varepsilon<2$. We show here our data for $y=t / s=4$, but the same behavior is observed for other values of $t / s$ accessed in this study, namely, $2 \leq t / s \leq 10$. However, closer inspection also shows that superuniversality is no longer true for relatively small spatial distances $|\boldsymbol{r}| / L(s) \lesssim 0.5$. This is shown in the insets of the panel (a) in Figs. 7 and 8. Indeed, systematic deviations are observed for small spatial distances, the largest deviations being observed for the autocorrelation and autoresponse functions with $|\boldsymbol{r}|=0$. The value of $|\boldsymbol{r}| / L(s)$ where the deviations set in seems to depend slightly on the value of $s$, but a larger range of $s$ values than that accessed in the present study is needed for a more quantitative discussion of this point. That means that although dynamical scaling does hold true even down to the autocorrelators and autoresponses, correlators and responses taken over a spatial dis-
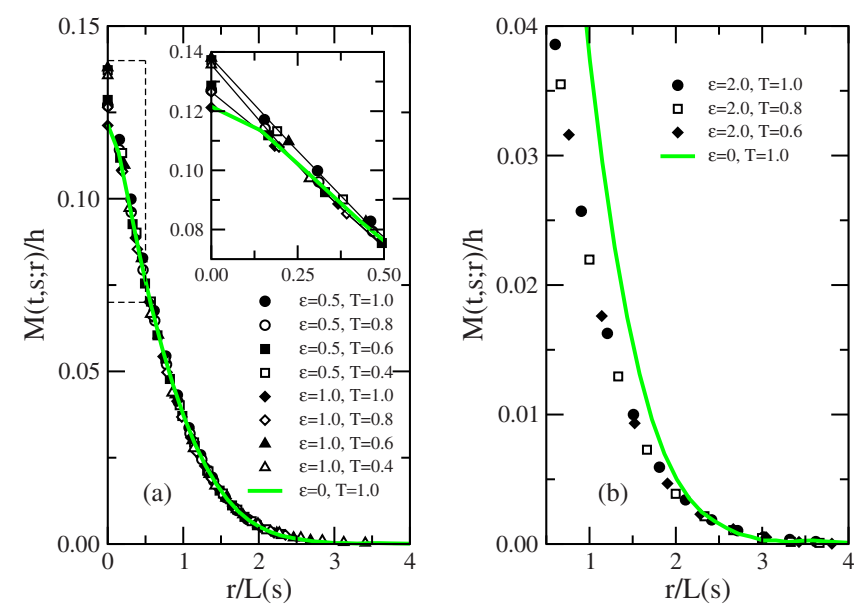

FIG. 8. (Color online) Test of the superuniversality of the twotime thermoremanent magnetization with $y=t / s=4$ and analogous to Fig. 6. The data shown are for $s=100$. In order to compare the data for different $\varepsilon$ and $T$, we have to take care of the fact that the response contains a nonuniversal multiplicative factor. The data have been multiplied by a constant in such a way that the different curves coincide with the curve for $\varepsilon=0$ and $T=1$ for large values of $\boldsymbol{r} / L(s)$. The gray curves show the data of the pure system. The inset in panel (a) is a magnification of the area within the dashed box. The black lines are guides for the eyes.

tance of at least a typical cluster size $L(t)$ show yet a larger degree of universality.

This requirement appears to be consistent with the known numerical values of the exponents $\lambda_{C} / z$ and $\lambda_{R} / z$ as listed in Table I. Superuniversality at $\boldsymbol{r}=\mathbf{0}$ would have required that their values should have been equal to those of the pure case $\varepsilon=0$, but we have rather seen that they depend on $\varepsilon$ and $T$.

On the other hand, the case $\varepsilon=2$ again stands apart, as we illustrate in the panels (b) in both Figs. 7 and 8. Comparing the data for $\varepsilon=2$ with the scaling functions found for $0 \leq \varepsilon$ $<2$ [see panels (a)], we find small but systematic deviations. We stress that although these deviations are not very large, they are well outside the error bars of our high-quality data. As for the single-time correlator, it remains a possibility that for enormous times there might occur a crossover to the scaling function of the pure case, but the relevant crossover time $t_{\times}$is far larger than the time scales reached by our simulation.

\section{DISCUSSION}

In this work, we have studied the nonequilibrium scaling behavior of a disordered Ising model without frustration in an attempt to appreciate better the role of disorder by considering its effects in a system which is no longer identical to a pure system but which yet does not show the full complexity of a spin glass. Our conclusions are as follows:

(1) When quenched to a temperature $T<T_{c}$ from a totally disordered state, the two-dimensional bond-disordered Ising model undergoes phase-ordering kinetics. The typical length scale $L(t) \sim t^{1 / z}$ of the ordered domains scales algebraically with time, where the dynamical exponent $z=z(\varepsilon / T)$ depends 
continuously on the dimensionless ratio $\varepsilon / T$ of the control parameters.

Quantitatively, this dependence can be read off from Fig. 2 and does not agree with earlier proposals of a linear relation such as in Eq. (5). We have seen that this value of $z$ correctly describes the dynamical scaling of not only singletime correlators but also of the two-time correlators and responses as well.

(2) Our data are completely compatible with simple aging.

(3) While the nonequilibrium exponent $b=0$ of the correlation function is unmodified with respect to pure phaseordering systems, the nonequilibrium exponent $a$ which describes the scaling of the response function is no longer simply related to the dynamical exponent $z$; see Table I and Fig. 2.

We propose to account for this finding in terms of a postulated fractal structure of the domain walls, which has led us to Eq. (11). Further tests of this idea would be welcome.

The autocorrelation and autoresponse exponents $\lambda_{C}$ $=\lambda_{C}(\varepsilon / T)$ and $\lambda_{R}=\lambda_{R}(T, \varepsilon)$ are distinct from each other, in contrast to the pure case.

(4) Our data suggest that the form of the scaling functions only depends on the dimensionless ratio $\varepsilon / T$. It remains to be seen to what extent this observation can be extended to different systems.

(5) In general, our data appear to confirm the superuniversality hypothesis that is when all length scales are expressed in terms of $L(t)$, the form of the scaling function is independent of both the disorder $\varepsilon$ and the temperature $T$.
However, we have also found two important qualifications:

(a) Superuniversality does not hold for sufficiently small spatial distances $|\boldsymbol{r}| / L(t) \lesssim 0.5$.

(b) For $\varepsilon=2$, although we find throughout a similar data collapse, the form of the scaling functions no longer coincides with the ones of the pure case. It is not understood whether the data presented here should be viewed as giving evidence for a distinct superuniversality class or else if there is a crossover to the scaling functions of the pure scale at time scales much larger than the ones reached in our study.

A better understanding of superuniversality will require an explanation of these conditions. What can these findings tell us on the behavior of real materials? Indeed, it has been shown recently, in a comparative study of the threedimensional random-field Ising model and the threedimensional Edwards-Anderson spin glass ${ }^{21}$ that superuniversality is apparently satisfied in the former case (in which the disorder is "weak" such that the ground state is still ferromagnetically ordered) while in the latter it is not. ${ }^{45}$ Our own result is in qualitative agreement with this, but it raises the question how to explain the celebrated universality of the scaling functions for the linear response found in largely different materials. ${ }^{1}$

\section{ACKNOWLEDGMENTS}

We thank Leticia Cugliandolo for useful discussions. The simulations were done on Virginia Tech's System X. M.H. thanks H. Park for kind hospitality at the KIAS Seoul, where the writing up of this work was finished.

*Also at Laboratoire associé au CNRS UMR 7556.

${ }^{1}$ L. C. E. Struik, Physical Ageing in Amorphous Polymers and Other Materials (Elsevier, Amsterdam, 1978).

${ }^{2}$ L. F. Cugliandolo, in Slow Relaxation and Non equilibrium Dynamics in Condensed Matter, edited by J.-L. Barrat, J. Dalibard, J. Kurchan, and M. V. Feigel'man (Springer, New York, 2003).

${ }^{3}$ E. Vincent, in Ageing and the Glass Transition, Springer Lecture Notes in Physics Vol. 716, edited by M. Henkel, M. Pleimling, and R. Sanctuary (Springer, Heidelberg, 2007), p. 7.

${ }^{4}$ A. J. Bray, Adv. Phys. 43, 357 (1994).

${ }^{5}$ O. Dauchot, in Ageing and the Glass Transition, Springer Lecture Notes in Physics Vol. 716, edited by M. Henkel, M. Pleimling, and R. Sanctuary (Springer, Heidelberg, 2007), p. 161.

${ }^{6}$ C. Godrèche and J.-M. Luck, J. Phys.: Condens. Matter 14, 1589 (2002).

${ }^{7}$ C. Chamon, L. F. Cugliandold, and H. Yoshino, J. Stat. Mech. (2006) P01006.

${ }^{8}$ C. Chamon and L. F. Cugliandolo, J. Stat. Mech. (2007) P07022.

${ }^{9}$ G. F. Mazenko, Nonequilibrium Statistical Mechanics (Wiley, New York, 2006), Chap. 11.

${ }^{10}$ A. Picone and M. Henkel, Nucl. Phys. B 688, 217 (2004).

${ }^{11}$ W. Zippold, R. Kühn, and H. Horner, Eur. Phys. J. B 13, 531 (2000).

${ }^{12}$ A. Andreanov and A. Lefèvre, Europhys. Lett. 76, 919 (2006).

${ }^{13}$ D. A. Huse and C. L. Henley, Phys. Rev. Lett. 54, 2708 (1985).

${ }^{14}$ Z. W. Lai, G. F. Mazenko, and O. T. Valls, Phys. Rev. B 37, 9481 (1988).

${ }^{15}$ D. S. Fisher and D. A. Huse, Phys. Rev. B 38, 373 (1988).

${ }^{16}$ A. J. Bray and K. Humayun, J. Phys. A 24, L1185 (1991).

${ }^{17}$ S. Puri, D. Chowdhuri, and N. Parekh, J. Phys. A 24, L1087 (1991).

${ }^{18}$ H. Hayakawa, J. Phys. Soc. Jpn. 60, 2492 (1991).

${ }^{19}$ T. Iwai and H. Hayakawa, J. Phys. Soc. Jpn. 62, 1583 (1993).

${ }^{20}$ B. Biswal, S. Puri, and D. Chowdhury, Physica A 229, 72 (1996).

${ }^{21}$ C. Aron, C. Chamon, L. F. Cugliandolo, and M. Picco, J. Stat. Mech. (2008) P05016.

${ }^{22}$ M. Henkel, Nucl. Phys. B 641, 405 (2002).

${ }^{23}$ M. Henkel and M. Pleimling, Phys. Rev. E 68, 065101(R) (2003).

${ }^{24}$ M. Henkel, A. Picone, and M. Pleimling, Europhys. Lett. 68, 191 (2004).

${ }^{25}$ E. Lorenz and W. Janke, Europhys. Lett. 77, 10003 (2007).

${ }^{26}$ M. Henkel and F. Baumann, J. Stat. Mech. (2007) P07015.

${ }^{27}$ R. Paul, S. Puri, and H. Rieger, Europhys. Lett. 68, 881 (2004).

${ }^{28}$ H. Rieger, G. Schehr, and R. Paul, Prog. Theor. Phys. 157, 111 (2005).

${ }^{29}$ R. Paul, S. Puri, and H. Rieger, Phys. Rev. E 71, 061109 (2005). 
${ }^{30}$ G. Schehr and P. Le Doussal, Europhys. Lett. 71, 290 (2005); G. Schehr and H. Rieger, Phys. Rev. B 71, 184202 (2005).

${ }^{31}$ M. Henkel and M. Pleimling, Europhys. Lett. 76, 561 (2006).

${ }^{32}$ A. Sicilia, J. J. Arenzon, A. J. Bray, and L. F. Cugliandolo, Europhys. Lett. 82, 10001 (2008).

${ }^{33}$ R. Paul, G. Schehr, and H. Rieger, Phys. Rev. E 75, 030104(R) (2007).

${ }^{34}$ A. Barrat, Phys. Rev. E 57, 3629 (1998).

${ }^{35}$ Indeed, if we take $\varepsilon=2$, then for $T \gtrsim 0.6$ notable finite-time corrections to simple aging are seen, which may be accounted for by including a finite-time correction term (Refs. 31 and 37). $C(t, s)=F_{C}(t / s)-s^{-b^{\prime}} G_{C}(t / s)$ and a new correction exponent $b^{\prime}>0$.

${ }^{36}$ If one uses a scaling form $C(t, s)=\mathcal{C}\left[(t-s) s^{-\mu}\right]$, it can be shown that the superaging case $\mu>1$ is incompatible with basic requirements from probability theory.

${ }^{37}$ J. Kurchan, Phys. Rev. E 66, 017101 (2002).

${ }^{38}$ M. Henkel and M. Pleimling, in Rugged Free-energy Landscapes: Common Computational Approaches in Spin Glasses, Structural Glasses, and Biological Macromolecules, Springer
Lecture Notes in Physics Vol. 736, edited by W. Janke (Springer, Heidelberg, 2007), p. 107.

${ }^{39}$ M. Henkel and M. Pleimling, Europhys. Lett. 69, 524 (2005).

${ }^{40}$ J.-P. Bouchaud, in Soft and Fragile Matter, edited by M. E. Cates and M. R. Evans (IOP, Bristol, 2000), p. 285.

${ }^{41}$ M. Henkel, M. Paeßens, and M. Pleimling, Europhys. Lett. 62, 664 (2003).

${ }^{42}$ A. Röthlein, F. Baumann, and M. Pleimling, Phys. Rev. E 74, 061604 (2006); A. Röthlein, F. Baumann, and M. Pleimling, ibid. 76, 019901(E) (2007).

${ }^{43}$ C. Yeung, M. Rao, and R. C. Desai, Phys. Rev. E 53, 3073 (1996).

${ }^{44}$ The consideration of the spatiotemporal response allows for a much more demanding test of dynamical scaling than is possible by merely considering the autoresponse alone. Our results hence strengthen the conclusions of a simple power-law scaling in the disordered Ising model reached earlier (Refs. 27, 29, and 31).

${ }^{45}$ It is conceivable that our finding of a distinct behavior for $\varepsilon=2$ might be a sort of borderline behavior when going from ferromagnets to frustrated magnet. 